\title{
The ISS 2B PVTCS Ammonia Leak: An Operational History
}

In 2006, the Photovoltaic Thermal Control System (PVTCS) for the International Space Station's 2B power channel began leaking ammonia at a rate of approximately $1.5 \mathrm{lbm} /$ year (out of a starting $~ 53 \mathrm{lbm}$ system ammonia mass). Initially, the operations strategy was "feed the leak," a strategy successfully put into action via Extra Vehicular Activity during the STS-134 mission. During this mission the system was topped off with ammonia piped over from a separate thermal control system. This recharge was to have allowed for continued power channel operation into 2014 or 2015, at which point another EVA would have been required. Without these periodic EVAs to refill the $2 \mathrm{~B}$ coolant system, the channel would eventually leak enough fluid as to risk pump cavitation and system failure, resulting in the loss of the 2B power channel - the most critical of the Space Station's 8 power channels.

In mid-2012, the leak rate increased to approximately 5lbm/year. Once discovered, an EVA was planned and executed within a 5 week timeframe to drastically alter the architecture of the PVTCS via connection to a dormant thermal control system not intended to be utilized as anything other than spare components. The purpose of this rerouting of the TCS was to increase system volume and to isolate the photovoltaic radiator, thought to be the likely leak source. This EVA was successfully executed on November $1^{\text {st }}, 2012$ and left the 2B PVTCS in a configuration where the system was now being adequately cooled via a totally different radiator than what the system was designed to utilize.

Unfortunately, data monitoring over the next several months showed that the isolated radiator was not leaking, and the system itself continued to leak steadily until May $9^{\text {th }}, 2013$. It was on this day that the ISS crew noticed the visible presence of ammonia crystals escaping from the $2 \mathrm{~B}$ channel's truss segment, signifying a rapid acceleration of the leak from $5 \mathrm{lbm} /$ year to $5 \mathrm{lbm} /$ day . Within 48 hours of the crew noticing the leak, an EVA was in progress to replace the coolant pump - the only other replaceable leak source.

This paper will explore the management of the 2B PVTCS leak from the operations perspective. It will discuss the methodology of performing the STS-134 refill, the considerations and contingency plans which went into the architectural overhaul of the system in 2012, and the unprecedented effort which went into the EVA response to the visible leak of May 2013. In particular the paper will focus on the techniques utilized by flight controllers to monitor the system health and to respond to such instances as the rapid May 2013 leak by putting the electrical system in a safe configuration for loss of cooling, and will use recorded telemetry of these events to describe system response to EVA crew and ground actions. It will discuss the innovative design for redundancy of the integrated truss structure's cooling systems which allowed for this leak to be managed with minimal impact to other ISS operations and electrical services, contrasted against the real unintended operations consequences of utilizing the flexibility of the spacecraft's design in this manner. The paper will discuss how the training of the crew and flight controller personnel has adapted to the changing architecture of the power system and the unpredictable nature of the $2 \mathrm{~B}$ leak. 